Stelle di confronto.

\begin{tabular}{|c|c|c|c|}
\hline$*$ & a I $88 \mathrm{I} .0$ & $\delta$ I 881.0 & Autorità \\
\hline I & $5^{\mathrm{h}} 3^{6^{\mathrm{mi}}} 4 \mathrm{O}^{\mathrm{s}} 92$ & $49^{\circ} 46^{\prime} 19^{\prime \prime} 6$ & 539 Sterne o Aurigae \\
\hline 2 & $545 \quad 57.40$ & $\begin{array}{lll}53 & 25 & 56.9\end{array}$ & Arg.-Oeltzen 6288 \\
\hline 3 & $9.20 \quad 0.22$ & 8 I 5 I 0.8 & 539 Sterne I H. Drac: \\
\hline 4 & - & - & DM. $+82^{0} 338$ \\
\hline 5 & I 2 I 242.8 & $\begin{array}{lll}8 I & 26 & \text { I } 8.4\end{array}$ & Rerlhill Cat. I 828 \\
\hline 6 & $12 \quad 1942.1$ & 812910.2 & Redhill Cat. I 845 \\
\hline 7 & $1230 \quad 32.17$ & $80 \quad 54 \quad 25.7$ & Redhill Cat. I 868 \\
\hline 8 & $1246 \quad 10.06$ & $\begin{array}{lll}81 & 3 & 19.8\end{array}$ & Redhill Cat. 1907 \\
\hline 9 & - & - & DM. $+80^{0} 395$ \\
\hline 10 & $\ldots$ & - & $\therefore+800398$ \\
\hline II & - & - & $\Rightarrow+80^{\prime} 404$ \\
\hline 12 & - & $\cdots$ & $"+80^{\circ} 409$ \\
\hline I 3 & - & - & $\Rightarrow+79^{\circ} 424$ \\
\hline
\end{tabular}

\begin{tabular}{|c|c|c|c|}
\hline$*$ & a 1881.0 & $\delta 1881.0$ & Autorità \\
\hline I 4 & I $3^{\mathrm{h}} 25^{\mathrm{m}} 57^{\mathrm{s}} 32$ & $79^{\prime \prime}$ I $5^{\prime} 31^{\prime \prime} 7$ & Greenwich I 864 \\
\hline 15 & - & - & DM. $+79^{\circ} 430$ \\
\hline 16 & — & 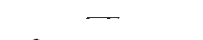 & $\nabla+78^{0} 468$ \\
\hline I 7 & 14436.60 & $78 \quad 39 \quad$ I 3.7 & Arg.-Oeltzen N. 14355 \\
\hline I 8 & $14 \quad 9 \quad 19.99$ & $\begin{array}{lll}78 & 6 & 24.2\end{array}$ & 539 Sterne 4 Urs. Mj. \\
\hline I9 & $1425 \quad 59.80$ & $77 \quad 20 \quad 32.0$ & Groombridge 2126 \\
\hline 20 & $1432 \quad 1.42$ & 515.2 & Groombridge 2 I 40 \\
\hline
\end{tabular}

La lettera T significa Tarchini, la lettera M: Millo. sevich: quest' ultimo fece tutte le riduzioni. Non si ridussero le stelle della Durchmusterung.

Roma 23. Agosto I8 I.

Pietro Tacchini.

\title{
Elemente und Ephemeride des Cometen e 1881.
}

Von Herrn Dr. H. Oppenheim.

Aus den Beobachtungen angestellt zu Paris I88 I October I Leipzig October I I und dem Mittel aus Kiel und Hamburg October I8, habe ich folgende Elemente des Cometen berechnet:

$$
\begin{aligned}
& 7=188 \mathrm{I} \text { September } 13.8288 \mathrm{~m} . \mathrm{Zt} \text {. Berlin. } \\
& \left.x-8=9^{\circ} 8^{\prime} 26^{\prime \prime}\right) \\
& s=26923 \quad 38\} \text { m.Aeq. I } 88 \text { I.o. } \\
& i=\text { II3 } 46 \quad 34 \\
& \log q=9.69454
\end{aligned}
$$

Zwei Elementensysteme, die ich aus den Beobachtungen: Wien October 8, Leipzig October I I und I3, als auch aus Paris October I, Wien October 8,

\begin{tabular}{|c|c|c|c|c|c|}
\hline $\begin{array}{l}2^{\mathrm{h}} \mathrm{m} . \mathrm{Zt} . \\
\text { Berlin }\end{array}$ & A.R & Decl. & $\log r$ & $\log A$ & $\mathrm{H}$ \\
\hline I $88 \mathrm{I}$ & & & & & \\
\hline Oct. 26 & I $3^{\text {h }} 50^{\mathrm{m}} 55^{\mathrm{s}}$ & $+28^{0} 9^{\prime} 9$ & 0.0284 & 0.2026 & 0.29 \\
\hline 27 & $51 \quad 17$ & $28 \quad 39.7$ & & & \\
\hline 28 & $5 \mathrm{I} 40$ & 299.5 & & & \\
\hline 29 & I3 52 & -2939.2 & & & \\
\hline
\end{tabular}
Berlin October 16 erhalten hatte, erwiesen sich als un-

\begin{tabular}{|c|c|c|c|c|c|}
\hline $\begin{array}{c}\mathrm{I} 2 \mathrm{~b} \\
\text { Berlin }\end{array}$ & A.R & Decl. & $\log r$ & $\log A$ & $\mathrm{H}$ \\
\hline $188 \mathrm{I}$ & & & & & \\
\hline Oct. 30 & I $3^{\mathrm{h}} 52^{\mathrm{m}} 24^{\mathrm{s}}$ & $+30^{\circ} 8^{\prime} 8$ & 0.0555 & 0.2043 & 0.25 \\
\hline $3 I$ & 5245 & $30 \quad 38.4$ & & & \\
\hline Nov. I & 536 & $3 \mathrm{I} \quad 8 . \mathrm{I}$ & & & \\
\hline 2 & 5326 & 3 I 37.9 & & & \\
\hline 3 & 5347 & $32 \quad 7.9$ & 0.0809 & 0.2051 & 0.22 \\
\hline 4 & 547 & $3238 . I$ & & & \\
\hline 5 & 5428 & 338.5 & & & \\
\hline 6 & 5448 & $33 \quad 39.2$ & & & \\
\hline 7 & 558 & 3410.1 & 0.1047 & 0.2048 & 0.20 \\
\hline 8 & 5528 & $3441 \cdot 3$ & & & \\
\hline 9 & 5549 & $35 \quad 12.7$ & & & \\
\hline IO & 56 & $3544 \cdot 5$ & & & \\
\hline I I & $56 \quad 29$ & $36 \quad 16.6$ & 0.1270 & 0.2038 & 0.18 \\
\hline I 2 & 5648 & $3649 . I$ & & & \\
\hline 13 & $57 \quad 7$ & 3721.9 & & & \\
\hline I 4 & 5726 & 3755.2 & & & \\
\hline I 5 & I3 5745 & +3828.9 & o. 1480 & 0.2021 & 0.16 \\
\hline
\end{tabular}
richtig, da die bei beiden Berechnungen benutzte Wiener Beobachtung mit einem bedeutenden Fehler behaftet scheint.
Die Helle fiir October I ist $=$ I gesetzt.

\section{H. Oppenheim.}

Be m e rk u n : Nach einer Nittheilung von Herrn J. Palisa an Dr. Oppenheim lautet die Declination der Wiener Beobachtung Oct. 8 :

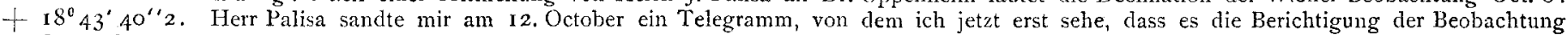

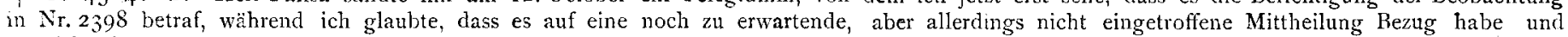
s) nicht den beabsichtigten Gebrauch davon machte. $K r$.

\section{Osservazione della cometa Denning fatta al R. Osservatorio del Collegio Romano.}

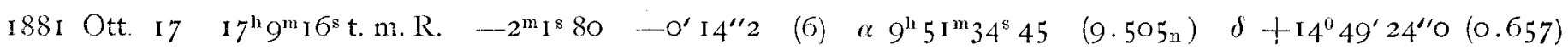
Stella di confr. 1881.0 a $9^{\mathrm{h}} 53^{\mathrm{m}} 33^{\mathrm{s}} 38+2^{\mathrm{s}} 87, \delta+\mathrm{I} 4^{0} 49^{\prime} 55^{\prime \prime} 4-\mathrm{I}^{\prime \prime} 2$ Weisse II $9^{\mathrm{h}} 1102$ E. Millosevich. 